\title{
Power series representations for bosonic effective actions
}

\section{Journal Article}

Author(s):

Balaban, Tadeusz; Feldman, Joel; Knörrer, Horst; Trubowitz, Eugene

Publication date:

2009

Permanent link:

https://doi.org/10.3929/ethz-b-000015360

Rights / license:

In Copyright - Non-Commercial Use Permitted

Originally published in:

Journal of Statistical Physics 134(5-6), https://doi.org/10.1007/s10955-008-9634-8 


\title{
Power Series Representations for Bosonic Effective Actions
}

\author{
Tadeusz Balaban · Joel Feldman · Horst Knörrer • \\ Eugene Trubowitz
}

Received: 21 July 2008 / Accepted: 10 October 2008 / Published online: 3 December 2008

(C) Springer Science+Business Media, LLC 2008

\begin{abstract}
We develop a power series representation and estimates for an effective action of the form

$$
\ln \frac{\int e^{f(\phi, \psi)} d \mu(\phi)}{\int e^{f(\phi, 0)} d \mu(\phi)}
$$

Here, $f(\phi, \psi)$ is an analytic function of the real fields $\phi(\mathbf{x}), \psi(\mathbf{x})$ indexed by $\mathbf{x}$ in a finite set $X$, and $d \mu(\phi)$ is a compactly supported product measure. Such effective actions occur in the small field region for a renormalization group analysis. The customary way to analyze them is a cluster expansion, possibly preceded by a decoupling expansion. Using methods similar to a polymer expansion, we estimate the power series of the effective action without introducing an artificial decomposition of the underlying space into boxes.
\end{abstract}

Keywords Polymer expansion · Renormalization group

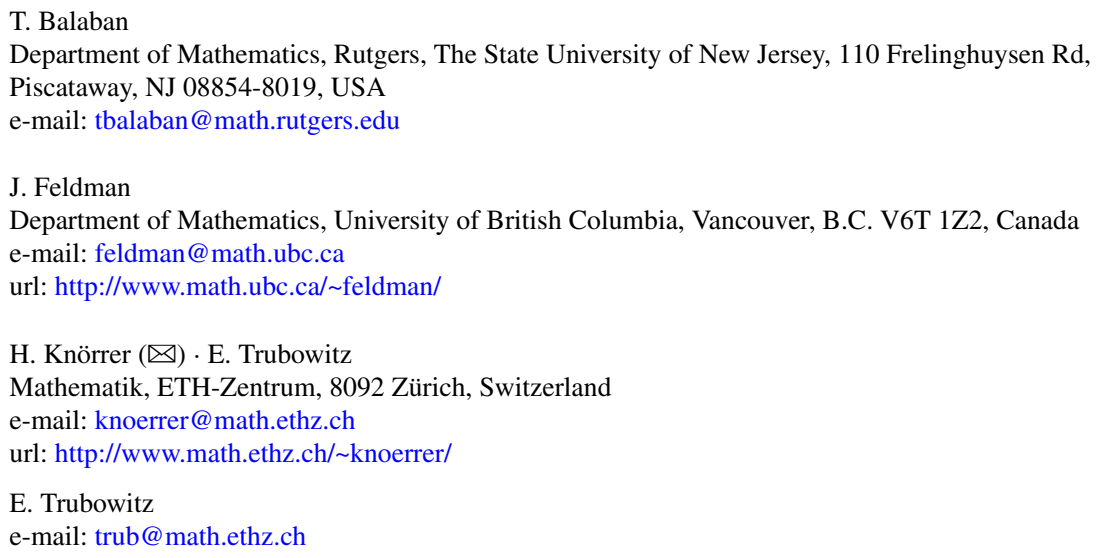




\section{Introduction}

We would like to propose a tool for use in the construction of certain bosonic field theories. To provide a context for the application of this tool, we start with a very schematic description of a typical Wilson style renormalization group construction.

One first expresses, formally, all quantities of interest as functional integrals like

$$
\mathcal{G}(\Psi)=\ln \frac{\int e^{\mathcal{A}(\Psi, \Phi)} d \mu(\Phi)}{\int e^{\mathcal{A}(0, \Phi)} d \mu(\Phi)}
$$

All of the correlation functions, for example, may be expressed in terms of derivatives of $\mathcal{G}(\Psi)$ with respect to the source field $\Psi$. The integration field $\Phi$ contains infinitely many degrees of freedom. But some of those degrees of freedom are more important than others. Therefore one factors the measure $d \mu(\Phi)=\prod_{\ell=1}^{\infty} d \mu_{\ell}\left(\varphi_{\ell}\right)$, with the less important degrees of freedom having smaller index $\ell$, and expresses

$$
\mathcal{G}(\Psi)=\ln \frac{\int e^{\mathcal{A}\left(\Psi, \varphi_{1}, \varphi_{2}, \ldots\right)} \prod_{\ell=1}^{\infty} d \mu_{\ell}\left(\varphi_{\ell}\right)}{\int e^{\mathcal{A}\left(0, \varphi_{1}, \varphi_{2}, \ldots\right)} \prod_{\ell=1}^{\infty} d \mu_{\ell}\left(\varphi_{\ell}\right)}
$$

Now one performs one integral at a time. Precisely, define the "effective action at scale $n$ " to be

$$
\mathcal{A}_{n}\left(\Psi, \varphi_{n+1}, \varphi_{n+2}, \ldots\right)=\ln \frac{\int e^{\mathcal{A}\left(\Psi, \varphi_{1}, \varphi_{2}, \ldots\right)} \prod_{\ell=1}^{n} d \mu_{\ell}\left(\varphi_{\ell}\right)}{\int e^{\mathcal{A}\left(0, \varphi_{1}, \ldots, \varphi_{n}, 0, \ldots\right)} \prod_{\ell=1}^{n} d \mu_{\ell}\left(\varphi_{\ell}\right)}
$$

Then we have the recursion relation

$$
\mathcal{A}_{n}(\psi)=\ln \frac{\int e^{\mathcal{A}_{n-1}(\psi, \phi)} d \mu_{n}(\phi)}{\int e^{\mathcal{A}_{n-1}(0, \phi)} d \mu_{n}(\phi)}
$$

where $\phi=\varphi_{n}$ and $\psi=\left(\Psi, \varphi_{n+1}, \varphi_{n+2}, \ldots\right)$.

Typically, the total contribution arising from large $\phi$ fields, for example field configurations with $\phi$ or appropriate derivatives large, is very small, reminiscent of large deviations in probability theory. On the other hand, contributions arising from integrals over regions where $\phi$ is small are physically important and must be analyzed in some detail. This "smallfield part" is extracted by replacing the measure $d \mu_{n}(\phi)$ by a measure $d \tilde{\mu}_{n}(\phi)$ that is supported on fields that obey (model and scale dependent) smallness conditions. Standard tools to prove the existence and control properties of

$$
\ln \frac{\int e^{\mathcal{A}_{n-1}(\psi, \phi)} d \tilde{\mu}_{n}(\phi)}{\int e^{\mathcal{A}_{n-1}(0, \phi)} d \tilde{\mu}_{n}(\phi)}
$$

are polymer expansions $[5,8-10]$. In the case that the actions are real analytic functions of the fields, we propose a related but different approach.

To avoid technical details, we assume that the fields are defined on a finite set $X$ (that is, the fields are elements of $\mathbb{R}^{X}$ ). Think of $X$ as an approximation to space, which is, for example, $\mathbb{Z}^{d}$ or $\mathbb{R}^{d}$. Fix a normalized measure $d \mu_{0}(t)$ on $\mathbb{R}$ that is supported in $|t| \leq r$ for some constant $r$. Thus

$$
\int|t|^{k} d \mu_{0}(t) \leq r^{k} \quad \text { for all } k \in \mathbb{N}
$$


We endow $\mathbb{R}^{X}$ with the ultralocal product measure

$$
d \mu(\phi)=\prod_{\mathbf{x} \in X} d \mu_{0}(\phi(\mathbf{x}))
$$

Furthermore, we consider functions $f(\psi ; \phi)$ that are analytic on a neighbourhood of the origin in $\mathbb{R}^{X} \times \mathbb{R}^{X}$ (the space of all pairs $(\psi, \phi)$ of fields). We think of $f(\psi ; \phi)$ as playing the role of $\mathcal{A}_{n-1}(\psi, \phi)$ in (1.1). In this note, we introduce norms for such functions that ensure the existence and analyticity of

$$
g(\psi)=\ln \frac{\int e^{f(\psi ; \phi)} d \mu(\phi)}{\int e^{f(0 ; \phi)} d \mu(\phi)}
$$

whenever the norm of $f(\psi ; \phi)$ is small enough. The norms are based on the power series expansion

$$
f(\psi ; \phi)=\sum_{m, n \geq 0} \sum_{\substack{\mathbf{x}_{1}, \ldots, \mathbf{x}_{m} \in X \\ \mathbf{y}_{1}, \ldots, \mathbf{y}_{n} \in X}} a\left(\mathbf{x}_{1}, \ldots, \mathbf{x}_{m} ; \mathbf{y}_{1}, \ldots, \mathbf{y}_{n}\right) \psi\left(\mathbf{x}_{1}\right) \cdots \psi\left(\mathbf{x}_{m}\right) \phi\left(\mathbf{y}_{1}\right) \cdots \phi\left(\mathbf{y}_{n}\right)
$$

of $f$ around the origin in $\mathbb{R}^{X} \times \mathbb{R}^{X}$. Here the coefficients are chosen to be invariant under permutations of $\mathbf{x}_{1}, \ldots, \mathbf{x}_{m}$ and $\mathbf{y}_{1}, \ldots, \mathbf{y}_{n}$. In addition to the existence of the logarithm (1.4), we obtain estimates on the coefficients in the power series expansion

$$
g(\psi)=\sum_{m \geq 0} \sum_{\mathbf{x}_{1}, \ldots, \mathbf{x}_{m} \in X} b\left(\mathbf{x}_{1}, \ldots, \mathbf{x}_{m}\right) \psi\left(\mathbf{x}_{1}\right) \cdots \psi\left(\mathbf{x}_{m}\right)
$$

Here is a simple example of one of these norms. Fix $\kappa>0$ and define

$$
\begin{aligned}
\|f(\psi ; \phi)\| & =\sum_{m, n \geq 0} \kappa^{m}(4 r)^{n} \max _{\mathbf{x} \in X} \max _{1 \leq i \leq m+n} \sum_{\substack{\mathbf{x}_{1}, \ldots, \mathbf{x}_{m+n} \in X \\
\mathbf{x}_{i}=\mathbf{x}}}\left|a\left(\mathbf{x}_{1}, \ldots, \mathbf{x}_{m} ; \mathbf{x}_{m+1}, \ldots, \mathbf{x}_{m+n}\right)\right| \\
\|g(\psi)\| & =\sum_{m \geq 0} \kappa^{m} \max _{\mathbf{x} \in X} \max _{1 \leq i \leq m} \sum_{\substack{\mathbf{x}_{1}, \ldots, \mathbf{x}_{m} \in X \\
\mathbf{x}_{i}=\mathbf{x}}}\left|b\left(\mathbf{x}_{1}, \ldots, \mathbf{x}_{m}\right)\right|
\end{aligned}
$$

In a typical application, $X$ is, for example, a discrete torus approximating a lattice, and the coefficients $a, b$ are translation invariant. The maxima in the norms are introduced to break translation invariance.

The main result, Theorem 3.4 states that if $\|f\|<\frac{1}{16}$ then the logarithm $g(\psi)$ exists and

$$
\|g\| \leq \frac{\|f\|}{1-16\|f\|}
$$

We consider more general norms than those given above. See Definitions 2.6 and 3.1. In particular, when $X$ is a metric space, spatial exponential decay may be incorporated. See Definition 2.5.

The algebraic structure of the proof of Theorem 3.4 is similar to that of the construction of the logarithm using polymer expansions [10, Sect. V.7]. Expand $e^{f(\psi ; \phi)}$ as

$$
1+\sum_{k=1}^{\infty} \frac{1}{k !} \sum_{\substack{Z_{1}, \ldots, Z_{k} \subset X \\ \text { pairwise disjoint }}} A\left(Z_{1}\right) \cdots A\left(Z_{k}\right)
$$


where $A(Z)$ is the sum of all products of monomials in the power series expansion of $f$ for which

$\circ$ the union of the supports for the $\phi$ fields in the monomials ${ }^{1}$ involved equals $Z$, and

$\circ$ the intersection graph of the supports for the $\phi$ fields in the monomials involved is connected.

Since the measure $d \mu(\phi)$ is ultralocal,

$$
\int e^{f(\psi ; \phi)} d \mu(\phi)=1+\sum_{k=1}^{\infty} \frac{1}{k !} \sum_{\substack{Z_{1}, \ldots, Z_{k} \subset X \\ \text { pairwise disjoint }}} \Phi\left(Z_{1}\right) \cdots \Phi\left(Z_{k}\right)
$$

where $\Phi(Z)=\int A(Z) d \mu(\phi)$. If the "pairwise disjoint" condition were not there, the right hand side would be exactly $\exp \left\{\sum_{Z \subset X} \Phi(Z)\right\}$ and taking the logarithm would be trivial. We use a standard procedure, that appears in all derivations of cluster expansions, to treat the "pairwise disjoint" condition and get the representation for the logarithm.

The restriction to ultralocal measures (1.3) is too severe to be directly useful. In part (i) of Proposition 1, we discuss the behaviour of our norms under linear changes of variables that might be used to diagonalize the covariance of a measure of interest. Part (ii) of this proposition controls the behaviour of these norms under substitutions like $f(\psi, \phi)=$ $g(\psi+\phi)$ that occur in renormalization group steps.

We developed the methods described in this paper in order to apply them to the (time-) ultraviolet analysis of a model for a Bose gas. For these models we must deal with complex rather than real fields [1, 2]. In a more technical paper [3], we develop analogs of the results of this paper for complex fields, as well as extensions and generalizations that are adapted to the geometry and scales of a large field/small field analysis of many-boson systems. As a sample application of the tool proposed here, [4] contains a complete description of the pure small field part of the (time-)ultraviolet analysis of the partition function for such a Bose gas.

\section{Norms}

In this section we specify the precise class of norms that we will use. We start by introducing some notation that will allow us to write the Taylor expansion of an analytic function $f\left(\psi_{1}, \ldots, \psi_{s}\right)$ of $s$ fields in a compact form.

Definition 2.1 ( $n$-tuples)

(i) Let $n \in \mathbb{Z}$ with $n \geq 0$ and $\overrightarrow{\mathbf{x}}=\left(\mathbf{x}_{1}, \ldots, \mathbf{x}_{n}\right) \in X^{n}$ be an ordered $n$-tuple of points of $X$. We denote by $n(\overrightarrow{\mathbf{x}})=n$ the number of components of $\overrightarrow{\mathbf{x}}$. Set

$$
\phi(\overrightarrow{\mathbf{x}})=\phi\left(\mathbf{x}_{1}\right) \cdots \phi\left(\mathbf{x}_{n}\right)
$$

If $n(\overrightarrow{\mathbf{x}})=0$, then $\phi(\overrightarrow{\mathbf{x}})=1$. The support of $\overrightarrow{\mathbf{x}}$ is defined to be

$$
\operatorname{supp} \overrightarrow{\mathbf{x}}=\left\{\mathbf{x}_{1}, \ldots, \mathbf{x}_{n}\right\} \subset X
$$

\footnotetext{
${ }^{1}$ The support for the $\phi$ fields in the monomial $a(\overrightarrow{\mathbf{x}} ; \overrightarrow{\mathbf{y}}) \psi\left(\mathbf{x}_{1}\right) \cdots \psi\left(\mathbf{x}_{m}\right) \phi\left(\mathbf{y}_{1}\right) \cdots \phi\left(\mathbf{y}_{n}\right)$ in the power series expansion of $f$ is the set $\left\{\mathbf{y}_{1}, \ldots, \mathbf{y}_{n}\right\} \subset X$. 
(ii) For each $s \in \mathbb{N}$, we denote ${ }^{2}$

$$
\mathbf{X}^{(s)}=\bigcup_{n_{1}, \ldots, n_{s} \geq 0} X^{n_{1}} \times \cdots \times X^{n_{s}}
$$

The support of $\left(\overrightarrow{\mathbf{x}}_{1}, \ldots, \overrightarrow{\mathbf{x}}_{s}\right) \in \mathbf{X}^{(s)}$ is

$$
\operatorname{supp}\left(\overrightarrow{\mathbf{x}}_{1}, \ldots, \overrightarrow{\mathbf{x}}_{s}\right)=\bigcup_{j=1}^{s} \operatorname{supp}\left(\overrightarrow{\mathbf{x}}_{j}\right)
$$

If $\left(\overrightarrow{\mathbf{x}}_{1}, \ldots, \overrightarrow{\mathbf{x}}_{s-1}\right) \in \mathbf{X}^{(s-1)}$ then $\left(\overrightarrow{\mathbf{x}}_{1}, \ldots, \overrightarrow{\mathbf{x}}_{s-1},-\right)$ denotes the element of $\mathbf{X}^{(s)}$ having $n\left(\overrightarrow{\mathbf{x}}_{s}\right)=0$. That is, $X^{0}=\{-\}$ and $\phi(-)=1$.

(iii) We define the concatenation of $\overrightarrow{\mathbf{x}}=\left(\mathbf{x}_{1}, \ldots, \mathbf{x}_{n}\right) \in X^{n}$ and $\overrightarrow{\mathbf{y}}=\left(\mathbf{y}_{1}, \ldots, \mathbf{y}_{m}\right) \in X^{m}$ to be

$$
\overrightarrow{\mathbf{x}} \circ \overrightarrow{\mathbf{y}}=\left(\mathbf{x}_{1}, \ldots, \mathbf{x}_{n}, \mathbf{y}_{1}, \ldots, \mathbf{y}_{m}\right) \in X^{n+m}
$$

For $\left(\overrightarrow{\mathbf{x}}_{1}, \ldots, \overrightarrow{\mathbf{x}}_{s}\right),\left(\overrightarrow{\mathbf{y}}_{1}, \ldots, \overrightarrow{\mathbf{y}}_{s}\right) \in \mathbf{X}^{(s)}$

$$
\left(\overrightarrow{\mathbf{x}}_{1}, \ldots, \overrightarrow{\mathbf{x}}_{s}\right) \circ\left(\overrightarrow{\mathbf{y}}_{1}, \ldots, \overrightarrow{\mathbf{y}}_{s}\right)=\left(\overrightarrow{\mathbf{x}}_{1} \circ \overrightarrow{\mathbf{y}}_{1}, \ldots, \overrightarrow{\mathbf{x}}_{s} \circ \overrightarrow{\mathbf{y}}_{s}\right)
$$

\section{Definition 2.2 (Coefficient Systems)}

(i) A coefficient system of length $s$ is a function $a\left(\overrightarrow{\mathbf{x}}_{1}, \ldots, \overrightarrow{\mathbf{x}}_{s}\right)$ which assigns a number to each $\left(\overrightarrow{\mathbf{x}}_{1}, \ldots, \overrightarrow{\mathbf{x}}_{s}\right) \in \mathbf{X}^{(s)}$. It is called symmetric if, for each $1 \leq j \leq s, a\left(\overrightarrow{\mathbf{x}}_{1}, \ldots, \overrightarrow{\mathbf{x}}_{s}\right)$ is invariant under permutations of the components of $\overrightarrow{\mathbf{x}}_{j}$.

(ii) Let $f\left(\psi_{1}, \ldots, \psi_{s}\right)$ be a function which is defined and real analytic on a neighbourhood of the origin in $\mathbb{R}^{s|X|}$. Then $f$ has a unique expansion of the form

$$
f\left(\psi_{1}, \ldots, \psi_{s}\right)=\sum_{\left(\overrightarrow{\mathbf{x}}_{1}, \ldots, \overrightarrow{\mathbf{x}}_{s}\right) \in \mathbf{X}^{(s)}} a\left(\overrightarrow{\mathbf{x}}_{1}, \ldots, \overrightarrow{\mathbf{x}}_{s}\right) \psi_{1}\left(\overrightarrow{\mathbf{x}}_{1}\right) \cdots \psi_{s}\left(\overrightarrow{\mathbf{x}}_{s}\right)
$$

with $a\left(\overrightarrow{\mathbf{x}}_{1}, \ldots, \overrightarrow{\mathbf{x}}_{s}\right)$ a symmetric coefficient system. This coefficient system is called the symmetric coefficient system of $f$.

Definition 2.3 (Weight Systems) A weight system of length $s$ is a function which assigns a positive extended number $w\left(\overrightarrow{\mathbf{x}}_{1}, \ldots, \overrightarrow{\mathbf{x}}_{s}\right) \in(0, \infty]$ to each $\left(\overrightarrow{\mathbf{x}}_{1}, \ldots, \overrightarrow{\mathbf{x}}_{s}\right) \in \mathbf{X}^{(s)}$ and satisfies the following conditions:

(a) For each $1 \leq j \leq s, w\left(\overrightarrow{\mathbf{x}}_{1}, \ldots, \overrightarrow{\mathbf{x}}_{s}\right)$ is invariant under permutations of the components (b) of $\overrightarrow{\mathbf{x}}_{j}$.

$$
w\left(\left(\overrightarrow{\mathbf{x}}_{1}, \ldots, \overrightarrow{\mathbf{x}}_{s}\right) \circ\left(\overrightarrow{\mathbf{y}}_{1}, \ldots, \overrightarrow{\mathbf{y}}_{s}\right)\right) \leq w\left(\overrightarrow{\mathbf{x}}_{1}, \ldots, \overrightarrow{\mathbf{x}}_{s}\right) w\left(\overrightarrow{\mathbf{y}}_{1}, \ldots, \overrightarrow{\mathbf{y}}_{s}\right)
$$

for all $\left(\overrightarrow{\mathbf{x}}_{1}, \ldots, \overrightarrow{\mathbf{x}}_{s}\right),\left(\overrightarrow{\mathbf{y}}_{1}, \ldots, \overrightarrow{\mathbf{y}}_{s}\right) \in \mathbf{X}^{(s)}$ with $\operatorname{supp}\left(\overrightarrow{\mathbf{x}}_{1}, \ldots, \overrightarrow{\mathbf{x}}_{s}\right) \cap \operatorname{supp}\left(\overrightarrow{\mathbf{y}}_{1}, \ldots, \overrightarrow{\mathbf{y}}_{s}\right) \neq \emptyset$.

\footnotetext{
${ }^{2}$ We distinguish between $X^{n_{1}} \times \cdots \times X^{n_{s}}$ and $X^{n_{1}+\cdots+n_{s}}$. We use $X^{n_{1}} \times \cdots \times X^{n_{s}}$ as the set of possible arguments for $\psi_{1}\left(\overrightarrow{\mathbf{x}}_{1}\right) \cdots \psi_{S}\left(\overrightarrow{\mathbf{x}}_{S}\right)$, while $X^{n_{1}+\cdots+n_{s}}$ is the set of possible arguments for $\psi_{1}\left(\overrightarrow{\mathbf{x}}_{1} \circ \cdots \circ \overrightarrow{\mathbf{x}}_{S}\right)$, where $\circ$ is the concatenation operator of part (iii).
} 


\section{Example 2.4 (Weight Systems)}

(i) If $\kappa_{1}, \ldots, \kappa_{s}$ are functions from $X$ to $(0, \infty]$ (called weight factors) then

$$
w\left(\overrightarrow{\mathbf{x}}_{1}, \ldots, \overrightarrow{\mathbf{x}}_{s}\right)=\prod_{j=1}^{s} \prod_{\ell=1}^{n\left(\overrightarrow{\mathbf{x}}_{j}\right)} \kappa_{j}\left(\mathbf{x}_{j, \ell}\right)
$$

is a weight system of length $s$.

(ii) Let $d: X \times X \rightarrow \mathbb{R}_{\geq 0}$ be a metric. The length of a tree $T$ with vertices in $X$ is the sum of the lengths of all edges of $T$ (where the length of an edge is the distance between its vertices). For a subset $S \subset X$, denote by $\tau(S)$ the length of a shortest tree in $X$ whose set of vertices contains $S$. If $m \geq 0$, then

$$
w\left(\overrightarrow{\mathbf{x}}_{1}, \ldots, \overrightarrow{\mathbf{x}}_{s}\right)=e^{m \tau\left(\operatorname{supp}\left(\overrightarrow{\mathbf{x}}_{1}, \ldots, \overrightarrow{\mathbf{x}}_{s}\right)\right)}
$$

is a weight system of length $s$.

(iii) If $w_{1}\left(\overrightarrow{\mathbf{x}}_{1}, \ldots, \overrightarrow{\mathbf{x}}_{s}\right)$ and $w_{2}\left(\overrightarrow{\mathbf{x}}_{1}, \ldots, \overrightarrow{\mathbf{x}}_{s}\right)$ are two weight systems of length $s$ then

$$
w_{3}\left(\overrightarrow{\mathbf{x}}_{1}, \ldots, \overrightarrow{\mathbf{x}}_{s}\right)=w_{1}\left(\overrightarrow{\mathbf{x}}_{1}, \ldots, \overrightarrow{\mathbf{x}}_{s}\right) w_{2}\left(\overrightarrow{\mathbf{x}}_{1}, \ldots, \overrightarrow{\mathbf{x}}_{s}\right)
$$

is also a weight systems of length $s$.

Definition 2.5 Assume that $X$ is a metric space. Given constants $\kappa_{j} \in(0, \infty]$ for $j=$ $1, \ldots, s$ and a mass $m \geq 0$ we call

$$
w\left(\overrightarrow{\mathbf{x}}_{1}, \ldots, \overrightarrow{\mathbf{x}}_{s}\right)=e^{m \tau\left(\operatorname{supp}\left(\overrightarrow{\mathbf{x}}_{1}, \ldots, \overrightarrow{\mathbf{x}}_{s}\right)\right)} \prod_{j=1}^{s} \kappa_{j}^{n\left(\overrightarrow{\mathbf{x}}_{j}\right)}
$$

the weight system with mass $m$ that associates the constant weight factor $\kappa_{j}$ to the field $\psi_{j}$. It follows from Example 2.4 that these are indeed weight systems.

\section{Definition 2.6 (Norms)}

(i) Let $w$ be a weight system and $a$ a coefficient system of length $s$. We define the norm of $a$ with weight $w$ to be

$$
|a|_{w}=\sum_{n_{1}, \ldots, n_{s} \geq 0} \max _{\mathbf{x} \in X} \max _{\substack{1 \leq j \leq s \\ n_{j} \neq 0}} \max _{1 \leq i \leq n_{j}} \sum_{\substack{\left(\overrightarrow{\mathbf{x}}_{1}, \ldots, \overrightarrow{\mathbf{x}}_{s}\right) \in X^{n_{1}}\left(\mathbf{x}_{j}\right)_{j}=\mathbf{x} \times X^{n_{s}} \\\left(\mathbf{x}_{j}=\mathbf{x}\right.}} w\left(\overrightarrow{\mathbf{x}}_{1}, \ldots, \overrightarrow{\mathbf{x}}_{s}\right)\left|a\left(\overrightarrow{\mathbf{x}}_{1}, \ldots, \overrightarrow{\mathbf{x}}_{s}\right)\right|
$$

Here $\left(\overrightarrow{\mathbf{x}}_{j}\right)_{i}$ is the $i^{\text {th }}$ component of the $n_{j}$-tuple $\overrightarrow{\mathbf{x}}_{j}$. The term in the above sum with $n_{1}, \ldots, n_{s}=0$ is simply $w(-, \ldots,-)|a(-, \ldots,-)|$.

(ii) Let $w$ be a weight system and $f\left(\psi_{1}, \ldots, \psi_{s}\right)$ be a function which is defined and analytic on a neighbourhood of the origin in $\mathbb{R}^{s|X|}$. The norm, $\|f\|_{w}$ of $f$ with weight $w$ is defined $^{3}$ to be $|a|_{w}$ where $a$ is the symmetric coefficient system of $f$.

\footnotetext{
${ }^{3}$ This definition also applies when $f$ depends only on a subset of the variables $\psi_{1}, \ldots, \psi_{s}$.
} 
Remark 2.7 Let $a$ be a (not necessarily symmetric) coefficient system of length $s$ and

$$
f\left(\psi_{1}, \ldots, \psi_{s}\right)=\sum_{\left(\overrightarrow{\mathbf{x}}_{1}, \ldots, \overrightarrow{\mathbf{x}}_{s}\right) \in \mathbf{X}^{(s)}} a\left(\overrightarrow{\mathbf{x}}_{1}, \ldots, \overrightarrow{\mathbf{x}}_{s}\right) \psi_{1}\left(\overrightarrow{\mathbf{x}}_{1}\right) \cdots \psi_{s}\left(\overrightarrow{\mathbf{x}}_{s}\right)
$$

Then $\|f\|_{w} \leq|a|_{w}$ for any weight system $w$. We call $a$ a (not necessarily symmetric) coefficient system for $f$.

Remark 2.8 Our motivation for the norm of Definition 2.6 is the following. As in the introduction, write

$$
g(\psi)=\ln \frac{\int e^{f(\psi ; \phi)} d \mu(\phi)}{\int e^{f(0 ; \phi)} d \mu(\phi)}=\sum_{n \geq 0} \sum_{\mathbf{x}_{1}, \ldots, \mathbf{x}_{n} \in X} b\left(\mathbf{x}_{1}, \ldots, \mathbf{x}_{n}\right) \psi\left(\mathbf{x}_{1}\right) \cdots \psi\left(\mathbf{x}_{n}\right)
$$

Then $b\left(\mathbf{x}_{1}, \ldots, \mathbf{x}_{n}\right)$ is an $n$ point connected correlation function. If $w(\overrightarrow{\mathbf{x}})$ is the weight system with mass $m$ that associates the constant weight factor 1 to the field $\psi$ as in Definition 2.5, then

$$
\max _{\mathbf{x} \in X} \max _{1 \leq i \leq n} \sum_{\substack{\left(\mathbf{x}_{1}, \ldots, \mathbf{x}_{n}\right) \in X^{n} \\ \mathbf{x}_{i}=\mathbf{x}}} w\left(\mathbf{x}_{1}, \ldots, \mathbf{x}_{n}\right)\left|b\left(\mathbf{x}_{1}, \ldots, \mathbf{x}_{n}\right)\right|
$$

is a norm for the $n$ point connected correlation function which takes into account its (typical) translation invariance and tests for exponential decay with mass $m$. The norm $\|g\|_{w}$ is the sum over $n$ of the norms of all of the $n$ point connected correlation functions.

\section{The Logarithm}

Definition 3.1 A weight system of length 2 "gives weight at least $\rho$ to the last field" if

$$
w(\overrightarrow{\mathbf{x}} ; \overrightarrow{\mathbf{y}}) \geq \rho^{n(\overrightarrow{\mathbf{y}})} w(\overrightarrow{\mathbf{x}} ;-)
$$

for all $(\overrightarrow{\mathbf{x}}, \overrightarrow{\mathbf{y}}) \in X^{(2)}$.

Example 3.2 Assume that $X$ is a metric space. Given a constant $\kappa \in(0, \infty]$ and a mass $m \geq 0$, the weight system with mass $m$ that associates the constant weight factor $\kappa$ to the field $\psi$ and the constant weight factor $\rho$ to the field $\phi$ gives weight at least $\rho$ to the last field.

Example 3.3 Let $w$ be a weight system of length 2 that gives weight at least $r$ to the last field. Recall that $r$ is the radius of support for the measure $d \mu(\phi)$, defined in (1.3). If $h$ is an analytic function for which $h(0 ; \phi)$ is constant, ${ }^{4}$ then

$$
\left\|\int h(\psi ; \phi) d \mu(\phi)\right\|_{w} \leq\|h(\psi ; \phi)\|_{w}
$$

\footnotetext{
${ }^{4}$ To see the need for this restriction, consider $h(\psi ; \phi)=\sum_{\mathbf{x} \in X} \phi(\mathbf{x})^{2}$ and $w(\overrightarrow{\mathbf{x}} ; \overrightarrow{\mathbf{y}})=r^{n(\overrightarrow{\mathbf{y}})}$. Then $\left\|\int h d \mu\right\|_{w}=|X| \int|t|^{2} d \mu_{0}(t)$ while $\|h\|_{w}=r^{2}$, independent of $|X|$.
} 
Theorem 3.4 Let $w$ be a weight system of length 2 that gives weight at least $4 r$ to the last field. ${ }^{5}$ If $f(\psi ; \phi)$ obeys $\|f\|_{w}<\frac{1}{16}$, then there is a real analytic function $g(\psi)$ such that

$$
\frac{\int e^{f(\psi ; \phi)} d \mu(\phi)}{\int e^{f(0 ; \phi)} d \mu(\phi)}=e^{g(\psi)}
$$

and

$$
\|g\|_{w} \leq \frac{\|f\|_{w}}{1-16\|f\|_{w}}
$$

Proof Let $a(\overrightarrow{\mathbf{x}} ; \overrightarrow{\mathbf{y}})$ be the symmetric coefficient system for $f$. By factoring $e^{f(\psi ; 0)}$ out of the integral in the numerator of (3.1) and $e^{f(0 ; 0)}$ out of the integral in the denominator, we may assume that $f(\psi ; 0)=0$ so that $a(\overrightarrow{\mathbf{x}} ;-)=0$ for all $\overrightarrow{\mathbf{x}} \in X^{(1)}$.

We first introduce some shorthand notation.

- The bulk of this proof concerns the integral over $\phi$, with $\psi$ just being viewed as a parameter. For this reason we write

$$
a(\overrightarrow{\mathbf{y}})=\sum_{\overrightarrow{\mathbf{x}} \in X^{(1)}} a(\overrightarrow{\mathbf{x}} ; \overrightarrow{\mathbf{y}}) \psi(\overrightarrow{\mathbf{x}})
$$

With this notation

$$
f(\psi ; \phi)=\sum_{\overrightarrow{\mathbf{y}} \in X^{(1)}} a(\overrightarrow{\mathbf{y}}) \phi(\overrightarrow{\mathbf{y}})
$$

and

$$
\begin{aligned}
& e^{f(\psi ; \phi)}=\sum_{\ell=0}^{\infty} \frac{1}{\ell !} f(\psi ; \phi)^{\ell} \\
& =1+\sum_{\ell=1}^{\infty} \frac{1}{\ell !} \sum_{\substack{Z \subset X \\
Z \neq \emptyset}} \sum_{\substack{\overrightarrow{\mathbf{y}}_{1}, \ldots, \overrightarrow{\mathbf{y}}_{\ell} \in X^{(1)} \\
Z=\text { supp.y }_{1} \cup \cdots \overrightarrow{s u p p}_{\ell}}} a\left(\overrightarrow{\mathbf{y}}_{1}\right) \cdots a\left(\overrightarrow{\mathbf{y}}_{\ell}\right) \phi\left(\overrightarrow{\mathbf{y}}_{1}\right) \cdots \phi\left(\overrightarrow{\mathbf{y}}_{\ell}\right)
\end{aligned}
$$

since $a(\overrightarrow{\mathbf{y}})=0$ unless $n(\overrightarrow{\mathbf{y}}) \geq 1$.

In a typical application the coefficient $a(\overrightarrow{\mathbf{y}})$ has decay properties that tend to keep the components of $\overrightarrow{\mathbf{y}}$ (and the $\overrightarrow{\mathbf{x}}$ components hidden inside $a(\overrightarrow{\mathbf{y}})$ ) close together. We would like to see similar decay properties in the coefficients of $g$. In the expansion (3.3), the coefficient $a\left(\overrightarrow{\mathbf{y}}_{1}\right)$ keeps the components of $\overrightarrow{\mathbf{y}}_{1}$ close together, ..., the coefficient $a\left(\overrightarrow{\mathbf{y}}_{\ell}\right)$ keeps the components of $\overrightarrow{\mathbf{y}}_{\ell}$ close together. But there is no reason for the components of $\overrightarrow{\mathbf{y}}_{i}$ to be close to the components of $\overrightarrow{\mathbf{y}}_{j}$, unless, by coincidence, the supports of $\overrightarrow{\mathbf{y}}_{i}$ and $\overrightarrow{\mathbf{y}}_{j}$ happen to intersect. Hence we will keep careful track of when the supports of $\overrightarrow{\mathbf{y}}_{i}$ and $\overrightarrow{\mathbf{y}}_{j}$ happen to intersect. This leads us to the following definitions.

○ Let $X_{1}, \ldots, X_{\ell}$ be subsets of $X$. The incidence graph $G\left(X_{1}, \ldots, X_{\ell}\right)$ of $X_{1}, \ldots, X_{\ell}$ is the labelled graph with the set of vertices $\{1, \ldots, \ell\}$ and edges between $i \neq j$ whenever $X_{i} \cap X_{j} \neq \emptyset$. For $\overrightarrow{\mathbf{y}}_{1}, \ldots, \overrightarrow{\mathbf{y}}_{\ell} \in X^{(1)}$ we write $G\left(\overrightarrow{\mathbf{y}}_{1}, \ldots, \overrightarrow{\mathbf{y}}_{\ell}\right)$ for $G\left(\operatorname{supp} \overrightarrow{\mathbf{y}}_{1}, \ldots, \operatorname{supp} \overrightarrow{\mathbf{y}}_{\ell}\right)$.

\footnotetext{
5 There is nothing magical about the choice of " 4 " in "gives weight at least $4 r$ ". This " 4 " may be replaced by any number strictly larger than 1 if one adjusts the final bound appropriately.
} 
$\circ$ A collection $\overrightarrow{\mathbf{y}}_{1}, \ldots, \overrightarrow{\mathbf{y}}_{n} \in X^{(1)}$ is called connected if the incidence graph $G\left(\overrightarrow{\mathbf{y}}_{1}, \ldots, \overrightarrow{\mathbf{y}}_{n}\right)$ is connected. For a subset of $Z \subset X$ we denote by $\mathcal{C}(Z)$ the set of all ordered $n$-tuples $\left(\overrightarrow{\mathbf{y}}_{1}, \ldots, \overrightarrow{\mathbf{y}}_{n}\right), n \in \mathbb{N}$, that are connected and for which $Z=\operatorname{supp} \overrightarrow{\mathbf{y}}_{1} \cup \cdots \cup \operatorname{supp} \overrightarrow{\mathbf{y}}_{n}$. We call such an $n$-tuple a connected cover of $Z$.

We now group the vectors $\overrightarrow{\mathbf{y}}_{1}, \ldots, \overrightarrow{\mathbf{y}}_{\ell}$ appearing in (3.3) into intersecting "clusters". Given a subset $Z$ of $X$ and $\overrightarrow{\mathbf{y}}_{1}, \ldots, \overrightarrow{\mathbf{y}}_{\ell} \in X^{(1)}$ with $Z=\operatorname{supp} \overrightarrow{\mathbf{y}}_{1} \cup \cdots \cup \operatorname{supp} \overrightarrow{\mathbf{y}}_{\ell}$, there is a (unique, up to labelling) decomposition of $\{1, \ldots, \ell\}$ into pairwise disjoint subsets $I_{1}, \ldots, I_{n}$ and a decomposition of $Z$ into pairwise disjoint subsets $Z_{1}, \ldots, Z_{n}$ such that, for each $1 \leq j \leq n$, $\left(\overrightarrow{\mathbf{y}}_{i}, i \in I_{j}\right)$ is a connected cover of $Z_{j}$. This decomposition corresponds to the decomposition of $G\left(\overrightarrow{\mathbf{y}}_{1}, \ldots, \overrightarrow{\mathbf{y}}_{\ell}\right)$ into connected components. Therefore

$$
\begin{aligned}
& e^{f}=1+\sum_{\ell=1}^{\infty} \frac{1}{\ell !} \sum_{n=1}^{\ell} \frac{1}{n !} \sum_{\substack{Z_{1}, \ldots, Z_{n} \subset X \\
\text { pairwise disjoint } \\
\text { nonempty }}} \sum_{\substack{I_{1} \cup \ldots \cup I_{n}=\{1, \ldots, \ell\} \\
I_{1}, \ldots, I_{n} \text { pairwise disjoint }}} \sum_{\substack{\left.\overrightarrow{\mathbf{y}}_{1}, \ldots, \overrightarrow{\mathbf{y}}_{\ell}, i \in I_{j}\right) \in \mathcal{C}\left(Z_{j}\right) \\
\left(\mathbf{y}^{\prime}\right.}} a\left(\overrightarrow{\mathbf{y}}_{1}\right) \cdots a\left(\overrightarrow{\mathbf{y}}_{\ell}\right) \\
& \times \phi\left(\overrightarrow{\mathbf{y}}_{1}\right) \cdots \phi\left(\overrightarrow{\mathbf{y}}_{\ell}\right)
\end{aligned}
$$

The next step is to reduce the combinatorial redundancy in this formula. Fix, for the moment, pairwise disjoint nonempty subsets $Z_{1}, \ldots, Z_{n}$ of $X$ and $\ell \geq n$. Then

$$
\begin{aligned}
& \sum_{\substack{I_{1} \cup \ldots \cup I_{n}=\{1, \ldots, \ell\} \\
I_{1}, \ldots, I_{n} \text { pairwise disjoint }}} \sum_{\substack{\overrightarrow{\mathbf{y}}_{1}, \ldots, \overrightarrow{\mathbf{y}}_{\ell} \\
\left(\overrightarrow{\mathbf{y}}_{i}, i \in I_{j}\right) \in \mathcal{C}\left(Z_{j}\right)}} a\left(\overrightarrow{\mathbf{y}}_{1}\right) \cdots a\left(\overrightarrow{\mathbf{y}}_{\ell}\right) \phi\left(\overrightarrow{\mathbf{y}}_{1}\right) \cdots \phi\left(\overrightarrow{\mathbf{y}}_{\ell}\right) \\
& =\sum_{\substack{k_{1}, \ldots, k_{n} \geq 1 \\
k_{1}+\cdots+k_{n}=\ell}} \sum_{\substack{I_{1}, \ldots, I_{n} \subset\{1, \ldots, \ell\} \\
I_{1}, \ldots, I_{n} \text { pairwise disjoint } \\
\left|I_{j}\right|=k_{j}}} \sum_{\substack{\overrightarrow{\mathbf{y}}_{1}, \ldots, \overrightarrow{\mathbf{y}}_{\ell} \\
\left(\overrightarrow{\mathbf{y}}_{i}, i \in I_{j}\right) \in \mathcal{C}\left(Z_{j}\right)}} a\left(\overrightarrow{\mathbf{y}}_{1}\right) \cdots a\left(\overrightarrow{\mathbf{y}}_{\ell}\right) \phi\left(\overrightarrow{\mathbf{y}}_{1}\right) \cdots \phi\left(\overrightarrow{\mathbf{y}}_{\ell}\right) \\
& =\sum_{\substack{k_{1}, \ldots, k_{n} \geq 1 \\
k_{1}+\cdots+k_{n}=\ell}} \frac{\ell !}{k_{1} ! \cdots k_{n} !} \sum_{\substack{\left(\overrightarrow{\mathbf{y}}_{1}, \ldots, \overrightarrow{\mathbf{y}}_{k_{1}}\right) \in \mathcal{C}\left(Z_{1}\right)\\
}} a\left(\overrightarrow{\mathbf{y}}_{1}\right) \cdots a\left(\overrightarrow{\mathbf{y}}_{\ell}\right) \phi\left(\overrightarrow{\mathbf{y}}_{1}\right) \cdots \phi\left(\overrightarrow{\mathbf{y}}_{\ell}\right) \\
& { }_{\left(\overrightarrow{\mathbf{y}}_{\ell-k_{n}+1}, \ldots, \overrightarrow{\mathbf{y}}_{\ell}\right) \in \mathcal{C}\left(Z_{n}\right)} \\
& =\ell ! \sum_{\substack{k_{1}, \ldots, k_{n} \geq 1 \\
k_{1}+\cdots+k_{n}=\ell}} \prod_{j=1}^{n}\left\{\frac{1}{k_{j} !} \sum_{\left.\overrightarrow{\mathbf{y}}_{1, j}, \ldots, \vec{y}_{k_{j}, j}\right) \in \mathcal{C}\left(Z_{j}\right)} a\left(\overrightarrow{\mathbf{y}}_{1, j}\right) \cdots a\left(\overrightarrow{\mathbf{y}}_{k_{j}, j}\right) \phi\left(\overrightarrow{\mathbf{y}}_{1, j}\right) \cdots \phi\left(\overrightarrow{\mathbf{y}}_{k_{j}, j}\right)\right\}
\end{aligned}
$$

Inserting this into (3.4) and exchanging the order of the $n$ and $\ell$ sums, we have

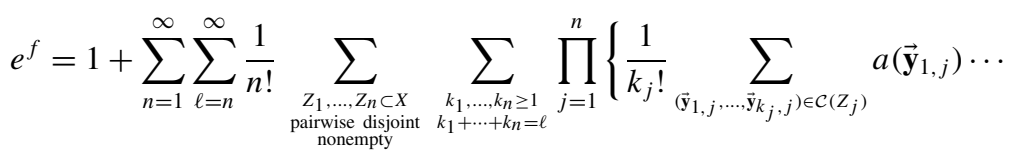

$$
\begin{aligned}
& \left.\times a\left(\overrightarrow{\mathbf{y}}_{k_{j}, j}\right) \phi\left(\overrightarrow{\mathbf{y}}_{1, j}\right) \cdots \phi\left(\overrightarrow{\mathbf{y}}_{k_{j}, j}\right)\right\} \\
& =1+\sum_{n=1}^{\infty} \frac{1}{n !} \sum_{\substack{Z_{1}, \ldots, Z_{n} \subset X \\
\text { pairwise disjoint } \\
\text { nonempty }}} \sum_{k_{1}, \ldots, k_{n} \geq 1} \prod_{j=1}^{n}\left\{\frac{1}{k_{j} !} \sum_{\left.\overrightarrow{\mathbf{y}}_{1, j}, \ldots, \overrightarrow{\mathbf{y}}_{k_{j}, j}\right) \in \mathcal{C}\left(Z_{j}\right)} a\left(\overrightarrow{\mathbf{y}}_{1, j}\right) \ldots\right. \\
& \left.\times a\left(\overrightarrow{\mathbf{y}}_{k_{j}, j}\right) \phi\left(\overrightarrow{\mathbf{y}}_{1, j}\right) \cdots \phi\left(\overrightarrow{\mathbf{y}}_{k_{j}, j}\right)\right\}
\end{aligned}
$$


As the measure $\mu$ factorizes with each factor normalized, and the different $Z_{j}$ 's are disjoint,

$$
\int \prod_{j=1}^{n}\left\{\phi\left(\overrightarrow{\mathbf{y}}_{1, j}\right) \cdots \phi\left(\overrightarrow{\mathbf{y}}_{k_{j}, j}\right)\right\} d \mu(\phi)=\prod_{j=1}^{n} \int \phi\left(\overrightarrow{\mathbf{y}}_{1, j}\right) \cdots \phi\left(\overrightarrow{\mathbf{y}}_{k_{j}, j}\right) d \mu(\phi)
$$

and we have

$$
\int e^{f(\psi ; \phi)} d \mu(\phi)=1+\sum_{n=1}^{\infty} \frac{1}{n !} \sum_{\substack{Z_{1}, \ldots, Z_{n} \subset X \\ \text { pairwise disjoint }}} \prod_{j=1}^{n} \Phi\left(Z_{j}\right)
$$

where, we define, for $\emptyset \neq Z \subset X$, the function $\Phi(Z)(\psi)$ by

$$
\Phi(Z)=\sum_{k=1}^{\infty} \frac{1}{k !} \sum_{\left(\overrightarrow{\mathbf{y}}_{1}, \ldots, \overrightarrow{\mathbf{y}}_{k}\right) \in \mathcal{C}(Z)} a\left(\overrightarrow{\mathbf{y}}_{1}\right) \cdots a\left(\overrightarrow{\mathbf{y}}_{k}\right) \int \phi\left(\overrightarrow{\mathbf{y}}_{1}\right) \cdots \phi\left(\overrightarrow{\mathbf{y}}_{k}\right) d \mu(\phi)
$$

and $\Phi(\emptyset)=0$.

We now deal with the "pairwise disjoint" condition in (3.5). If we define

$$
\zeta\left(Z, Z^{\prime}\right)= \begin{cases}0 & \text { if } Z \cap Z^{\prime} \neq \emptyset \\ 1 & \text { if } Z \text { and } Z^{\prime} \text { are disjoint }\end{cases}
$$

and $G_{n}=\left\{\{i, j\} \subset \mathbb{N}^{2} \mid 1 \leq i<j \leq n\right\}$ is the complete graph on $\{1, \ldots, n\}$, then

$$
\begin{aligned}
\int e^{f(\psi ; \phi)} d \mu(\phi) & =1+\sum_{n=1}^{\infty} \frac{1}{n !} \sum_{Z_{1}, \ldots, Z_{n} \subset X} \prod_{\{i, j\} \in G_{n}} \zeta\left(Z_{i}, Z_{j}\right) \prod_{j=1}^{n} \Phi\left(Z_{j}\right) \\
& =1+\sum_{n=1}^{\infty} \frac{1}{n !} \sum_{Z_{1}, \ldots, Z_{n} \subset X}\left(\sum_{g \subset G_{n}} \prod_{\{i, j\} \in g}\left(\zeta\left(Z_{i}, Z_{j}\right)-1\right)\right) \prod_{j=1}^{n} \Phi\left(Z_{j}\right)
\end{aligned}
$$

by the binomial expansion. Here, whenever a product $\prod_{\{i, j\} \in G_{n}}$ or $\prod_{\{i, j\} \in g}$ is empty, as is the case for $n=1$, it is given the value one. We may identify each $g \subset G_{n}$ with the labelled graph on the set of vertices $\{1, \ldots, n\}$ that has an edge joining vertex $i$ and vertex $j$ if and only if $\{i, j\} \in g$. Denote by $\mathcal{G}_{n}$ the set of all graphs (connected or not) on the set of vertices $\{1, \ldots, n\}$ that have at most one edge joining each pair of distinct vertices and no edges joining a vertex to itself. Define

$$
\rho\left(Z_{1}, \ldots, Z_{n}\right)= \begin{cases}1 & \text { if } n=1 \\ \sum_{g \in \mathcal{G}_{n}} \prod_{\{i, j\} \in g}\left(\zeta\left(Z_{i}, Z_{j}\right)-1\right) & \text { if } n \geq 2\end{cases}
$$

In this notation

$$
\int e^{f(\psi ; \phi)} d \mu(\phi)=1+\sum_{n=1}^{\infty} \frac{1}{n !} \sum_{Z_{1}, \ldots, Z_{n} \subset X} \rho\left(Z_{1}, \ldots, Z_{n}\right) \prod_{j=1}^{n} \Phi\left(Z_{j}\right)
$$

Now let $\mathcal{C}_{n} \subset \mathcal{G}_{n}$ be the set of all connected graphs on the set of vertices $\{1, \ldots, n\}$ that have at most one edge joining each pair of distinct vertices and no edges joining a vertex to itself. Set

$$
\rho^{T}\left(Z_{1}, \ldots, Z_{n}\right)= \begin{cases}1 & \text { if } n=1 \\ \sum_{g \in \mathcal{C}_{n}} \prod_{\{i, j\} \in g}\left(\zeta\left(Z_{i}, Z_{j}\right)-1\right) & \text { if } n \geq 2\end{cases}
$$


Note, in particular, that $\rho^{T}\left(Z_{1}, \ldots, Z_{n}\right)=0$ if $G\left(Z_{1}, \ldots, Z_{n}\right)$ is not connected (since, in this case, at least one factor of $\prod_{\{i, j\} \in g}\left(\zeta\left(Z_{i}, Z_{j}\right)-1\right)$ vanishes for each $\left.g \in \mathcal{C}_{n}\right)$. By a standard argument (see, for example [8, Theorem 2.17]),

$$
\ln \int e^{f} d \mu=\sum_{n=1}^{\infty} \frac{1}{n !} \sum_{Z_{1}, \ldots, Z_{n} \subset X} \rho^{T}\left(Z_{1}, \ldots, Z_{n}\right) \prod_{j=1}^{n} \Phi\left(Z_{j}\right)
$$

(By "ln" we just mean that the exponential of the right hand side is $\int e^{f} d \mu$.)

Let, for any connected graph $G \in \mathcal{C}_{n}$,

$$
t(G)= \begin{cases}1 & \text { if } n=1 \\ \sum_{\substack{g \in \mathcal{C}_{n} \\ g \subset G}}(-1)^{|g|} & \text { if } n>1\end{cases}
$$

The bound

$$
|t(G)| \leq \#\{\text { spanning trees in } G\}
$$

is due to Rota [7]. For a simple proof see [10, Theorem V.7.A.6]. If $G\left(Z_{1}, \ldots, Z_{n}\right)$ is connected, we have that

$$
\rho^{T}\left(Z_{1}, \ldots, Z_{n}\right)=\sum_{\substack{g \in \mathcal{C}_{n} \\ g \subset G\left(Z_{1}, \ldots, Z_{n}\right)}}(-1)^{|g|}=t\left(G\left(Z_{1}, \ldots, Z_{n}\right)\right)
$$

and hence

$$
\left|\rho^{T}\left(Z_{1}, \ldots, Z_{n}\right)\right| \leq \#\left\{\operatorname{tree} T \text { on }\{1, \ldots, n\}|| T \mid=n-1, T \subset G\left(Z_{1}, \ldots, Z_{n}\right)\right\}
$$

To get a, not necessarily symmetric, coefficient system for $\ln \int e^{f} d \mu$ above we first construct a coefficient system for each $\Phi(Z)$. For each $(\overrightarrow{\mathbf{x}}, \overrightarrow{\mathbf{y}}) \in X^{(2)}$, set

$$
\tilde{a}(\overrightarrow{\mathbf{x}} ; \overrightarrow{\mathbf{y}})=\sum_{k=1}^{\infty} \frac{1}{k !} \sum_{\substack{\left.\left(\overrightarrow{\mathbf{y}}_{1}, \ldots, \overrightarrow{\mathbf{y}}_{k}\right) \in \mathcal{C}(\text { supp }) \\ \overrightarrow{\mathbf{y}}_{1} \ldots \ldots \mathbf{y}_{k}=\overrightarrow{\mathbf{y}}\right)}} \sum_{\substack{\overrightarrow{\mathbf{x}}_{1}, \ldots, \overrightarrow{\mathbf{x}}_{k} \\ \mathbf{x}_{1} 0 \ldots \times \mathbf{x}_{k}=\overrightarrow{\mathbf{x}}}} a\left(\overrightarrow{\mathbf{x}}_{1} ; \overrightarrow{\mathbf{y}}_{1}\right) \cdots a\left(\overrightarrow{\mathbf{x}}_{k} ; \overrightarrow{\mathbf{y}}_{k}\right) \int \phi(\overrightarrow{\mathbf{y}}) d \mu(\phi)
$$

if $n(\overrightarrow{\mathbf{y}}) \geq 1$ and $\tilde{a}(\overrightarrow{\mathbf{x}} ; \overrightarrow{\mathbf{y}})=0$ if $n(\overrightarrow{\mathbf{y}})=0$. By (3.6) and (3.2),

$$
\Phi(Z)(\psi)=\sum_{\substack{(\overrightarrow{\mathbf{x}}, \overrightarrow{\mathbf{y}}) \in X(2) \\ \operatorname{supp} \overrightarrow{\mathbf{y}}=Z}} \tilde{a}(\overrightarrow{\mathbf{x}} ; \overrightarrow{\mathbf{y}}) \psi(\overrightarrow{\mathbf{x}})
$$

Therefore, by (3.7),

$$
\ln \int e^{f} d \mu=\sum_{\overrightarrow{\mathbf{x}} \in X^{(1)}} a^{\prime}(\overrightarrow{\mathbf{x}}) \psi(\overrightarrow{\mathbf{x}})
$$

where, for $\overrightarrow{\mathbf{x}} \in X^{(1)}$,

$$
a^{\prime}(\overrightarrow{\mathbf{x}})=\sum_{n=1}^{\infty} \frac{1}{n !} \sum_{\substack{\overrightarrow{\mathbf{x}}_{1}, \ldots, \overrightarrow{\mathbf{x}}_{n} \in X^{(1)} \\ \overrightarrow{\mathbf{x}}_{1} 0 \ldots \circ \mathbf{\mathbf { x }}_{n}=\mathbf{\mathbf { x }}}} \sum_{\overrightarrow{\mathbf{y}}_{1}, \ldots, \overrightarrow{\mathbf{y}}_{n} \in X^{(1)}} \rho^{T}\left(\operatorname{supp} \overrightarrow{\mathbf{y}}_{1}, \ldots, \operatorname{supp} \overrightarrow{\mathbf{y}}_{n}\right) \prod_{j=1}^{n} \tilde{a}\left(\overrightarrow{\mathbf{x}}_{j} ; \overrightarrow{\mathbf{y}}_{j}\right)
$$


Also

$$
g(\psi)=\ln \frac{\int e^{f(\psi ; \phi)} d \mu(\phi)}{\int e^{f(0 ; \phi)} d \mu(\phi)}=\sum_{\substack{\overrightarrow{\mathbf{x}} \in X^{(1)} \\ \overrightarrow{\mathbf{x}} \neq-}} a^{\prime}(\overrightarrow{\mathbf{x}}) \psi(\overrightarrow{\mathbf{x}})
$$

so that $a^{\prime}$, excluding the constant term $a^{\prime}(-)$, is a, not necessarily symmetric, coefficient system for $g$. By Remark 2.7,

$$
\|g\|_{w} \leq\left|a^{\prime}\right|_{w}
$$

We now bound the coefficient system $a^{\prime}(\overrightarrow{\mathbf{x}})$ for $g(\psi)$. Equation (3.10) expresses the output coefficients $a^{\prime}(\overrightarrow{\mathbf{x}})$ in terms of the intermediate coefficients $\tilde{a}(\overrightarrow{\mathbf{x}} ; \overrightarrow{\mathbf{y}})$. Equation (3.9), in turn, expresses the intermediate coefficients $\tilde{a}(\overrightarrow{\mathbf{x}} ; \overrightarrow{\mathbf{y}})$ in terms of the input coefficients $a(\overrightarrow{\mathbf{x}} ; \overrightarrow{\mathbf{y}})$. These formulae lead to the following bounds.

For each nontrivial $\overrightarrow{\mathbf{x}} \in X^{(1)}$, by (3.10) and (3.8),

$$
\begin{aligned}
& \left|a^{\prime}(\overrightarrow{\mathbf{x}})\right| \leq \sum_{n=1}^{\infty} \frac{1}{n !} \sum_{\substack{\overrightarrow{\mathbf{x}}_{1}, \ldots, \overrightarrow{\mathbf{x}}_{n} \in X^{(1)} \\
\overrightarrow{\mathbf{x}}_{1} \ldots \ldots \mathbf{x}_{n}=\mathbf{\mathbf { x }}}} \sum_{\overrightarrow{\mathbf{y}}_{1}, \ldots, \overrightarrow{\mathbf{y}}_{n} \in X^{(1)}} \sum_{\substack{T \text { spanning tree } \\
\text { for } G\left(\overrightarrow{\mathbf{y}}_{1}, \ldots, \overrightarrow{\mathbf{y}}_{n}\right)}} \prod_{j=1}^{n}\left|\tilde{a}\left(\overrightarrow{\mathbf{x}}_{j} ; \overrightarrow{\mathbf{y}}_{j}\right)\right|
\end{aligned}
$$

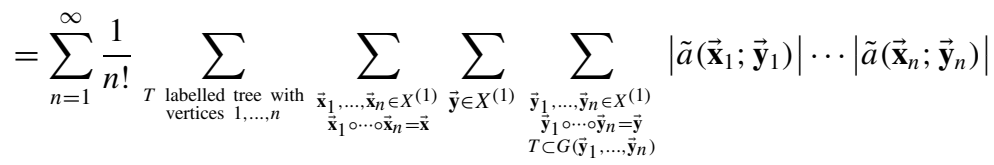

$$
\begin{aligned}
& =\sum_{n=1}^{\infty} \frac{1}{n !} \sum_{\substack{T \\
\text { labelled tree with } \\
\text { vertices } 1, \ldots, n}} \sum_{\overrightarrow{\mathbf{y}} \in X^{(1)}}|\tilde{a}|_{T}(\overrightarrow{\mathbf{x}} ; \overrightarrow{\mathbf{y}})
\end{aligned}
$$

where

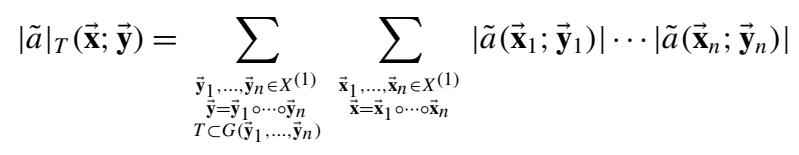

There is a similar bound for $\tilde{a}(\overrightarrow{\mathbf{x}} ; \overrightarrow{\mathbf{y}})$. Since, for every $\left(\overrightarrow{\mathbf{y}}_{1}, \ldots, \overrightarrow{\mathbf{y}}_{k}\right)$ contributing to (3.9), $G\left(\overrightarrow{\mathbf{y}}_{1}, \ldots, \overrightarrow{\mathbf{y}}_{k}\right)$ is connected and hence contains at least one tree, and since the measure $d \mu_{0}(\phi(\mathbf{x}))$ is normalized and supported on $[-r, r]$, we have

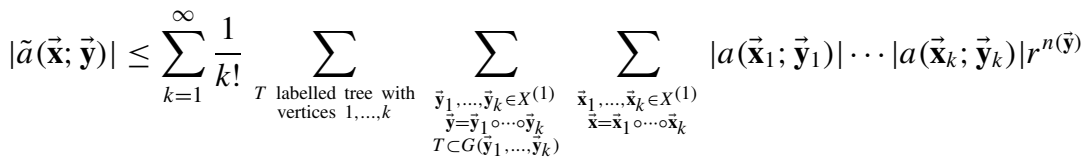

$$
\begin{aligned}
& =\sum_{k=1}^{\infty} \frac{1}{k !} \sum_{\substack{T \\
\text { labelled tree with } \\
\text { vertices } 1, \ldots, k}} r^{n(\overrightarrow{\mathbf{y}})}|a|_{T}(\overrightarrow{\mathbf{x}} ; \overrightarrow{\mathbf{y}})
\end{aligned}
$$

where

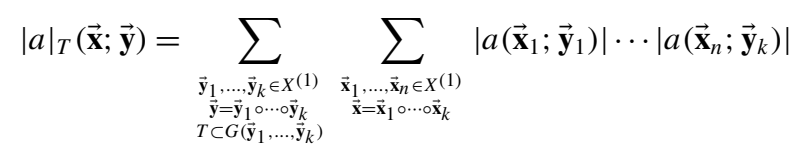


To complete the proof of Theorem 3.4, we use two lemmata. The first, Lemma 3.5, below, provides bounds on $|\tilde{a}|_{T}$ in terms of $\tilde{a}$ and on $|a|_{T}$ in terms of $a$. The second, Lemma 3.6, below, bounds the sums that result from the application of the first lemma.

We introduce, for each $\sigma>0$, the auxiliary weight system

$$
w_{\sigma}(\overrightarrow{\mathbf{x}} ; \overrightarrow{\mathbf{y}})=w(\overrightarrow{\mathbf{x}} ; \overrightarrow{\mathbf{y}})\left(\frac{\sigma}{4 r}\right)^{n(\overrightarrow{\mathbf{y}})}
$$

By parts (i) and (iii) of Example 2.4, $w_{\sigma}$ is indeed a weight system. Clearly $w_{4 r}=w$. Furthermore

$$
w(\overrightarrow{\mathbf{x}} ;-) \leq w_{1}(\overrightarrow{\mathbf{x}} ; \overrightarrow{\mathbf{y}})
$$

for all $(\overrightarrow{\mathbf{x}}, \overrightarrow{\mathbf{y}}) \in X^{(2)}$, by Definition 3.1, with $\rho=4 r$.

By (3.12), (3.14) and Lemma 3.5, with $\omega=w_{1}$ and $\omega^{\prime}=w_{2}$,

$$
\begin{aligned}
& \left|a^{\prime}-a^{\prime}(-)\right|_{w} \leq\left.\left.\sum_{n=1}^{\infty} \frac{1}{n !} \sum_{\substack{T \\
\text { labelled tree with } \\
\text { vertices } 1, \ldots, n}}|| \tilde{a}\right|_{T}\right|_{w_{1}}
\end{aligned}
$$

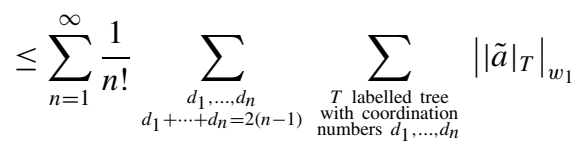

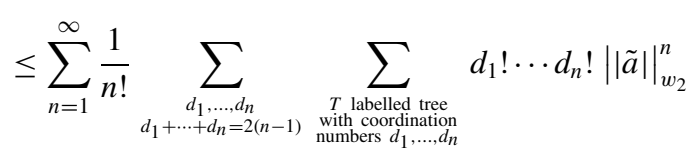

Now apply Lemma 3.6 with $\varepsilon=\|\left.\tilde{a}\right|_{w_{2}}=|\tilde{a}|_{w_{2}}$ and $v=1$ to get

$$
\left|a^{\prime}\right|_{w} \leq \frac{|\tilde{a}|_{w_{2}}}{1-8|\tilde{a}|_{w_{2}}}
$$

By construction, $\left.\left.\left|r^{n(\overrightarrow{\mathbf{y}})}\right| a\right|_{T}(\overrightarrow{\mathbf{x}} ; \overrightarrow{\mathbf{y}})\right|_{w_{2}}=\left.\left.|| a\right|_{T}\right|_{w_{2 r}}$. Hence, by (3.13) and Lemma 3.5, with $\omega=w_{2 r}$, followed by Lemma 3.6,

$$
\begin{aligned}
& |\tilde{a}|_{w_{2}} \leq\left.\left.\sum_{k=1}^{\infty} \frac{1}{k !} \sum_{\substack{T \\
\text { abelled tree with } \\
\text { vertices } 1, \ldots, k}}|| a\right|_{T}\right|_{w_{2 r}} \\
& \leq \sum_{k=1}^{\infty} \frac{1}{k !} \sum_{\substack{d_{1}, \ldots, d_{k} \\
d_{1}+\cdots+d_{k}=2(k-1)}} \sum_{\begin{array}{c}
T \text { labelled tree } \\
\text { with coordination } \\
\text { numbers } d_{1}, \ldots, d_{k}
\end{array}} d_{1} ! \cdots d_{k} !|a|_{w_{4 r}}^{k} \\
& \leq \frac{|a|_{w}}{1-8|a|_{w}}
\end{aligned}
$$

since $w_{4 r}=w$.

Combining (3.11), (3.15) and (3.16) yields

$$
\|g\|_{w} \leq \frac{|a|_{w}}{1-16|a|_{w}}=\frac{\|f\|_{w}}{1-16\|f\|_{w}}
$$


Lemma 3.5 Let $\omega$ be an arbitrary weight system of length 2 and define the weight system $\omega^{\prime}$ by

$$
\omega^{\prime}(\overrightarrow{\mathbf{x}} ; \overrightarrow{\mathbf{y}})=2^{n(\overrightarrow{\mathbf{y}})} \omega(\overrightarrow{\mathbf{x}} ; \overrightarrow{\mathbf{y}})
$$

Let $T$ be a labelled tree with vertices $1, \ldots, n$ and coordination numbers $d_{1}, \ldots, d_{n}$. Let $b$ be any (not necessarily symmetric) coefficient system of length 2 with $b(-;-)=0$. We define a new coefficient system $b_{T}$ by

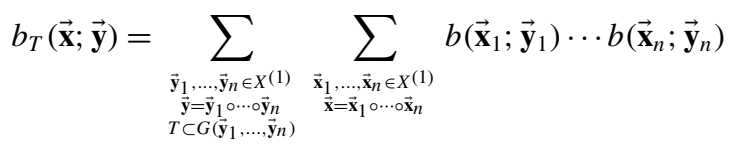

Then

$$
\left|b_{T}\right|_{\omega} \leq d_{1} ! \cdots d_{n} !|b|_{\omega^{\prime}}^{n}
$$

Proof For any pair $\vec{N}=\left(N_{1}, N_{2}\right)$ of nonnegative integers, let $b_{\vec{N}}(\overrightarrow{\mathbf{x}}, \overrightarrow{\mathbf{y}})$ denote the restriction of $b(\overrightarrow{\mathbf{x}} ; \overrightarrow{\mathbf{y}})$ to $(n(\overrightarrow{\mathbf{x}}), n(\overrightarrow{\mathbf{y}}))=\vec{N}$. That is,

$$
b_{\vec{N}}(\overrightarrow{\mathbf{x}} ; \overrightarrow{\mathbf{y}})= \begin{cases}b(\overrightarrow{\mathbf{x}} ; \overrightarrow{\mathbf{y}}) & \text { if } n(\overrightarrow{\mathbf{x}})=N_{1}, n(\overrightarrow{\mathbf{y}})=N_{2} \\ 0 & \text { otherwise }\end{cases}
$$

Then

$$
b_{T}=\sum_{\vec{N}^{(1)}, \ldots, \vec{N}^{(n)} \in \mathbb{N}_{0}^{2}} b_{\vec{N}^{(1)}, \ldots, \vec{N}^{(n)}}
$$

where

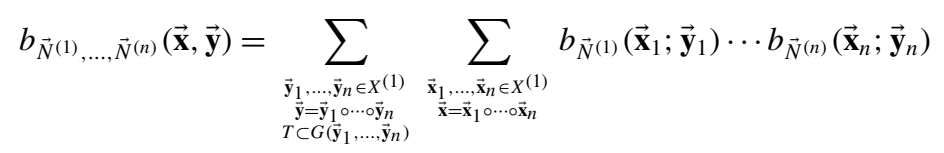

Since

$$
\left|b_{T}\right|_{\omega} \leq \sum_{\vec{N}^{(1)}, \ldots, \vec{N}^{(n)} \in \mathbb{N}_{0}^{2}}\left|b_{\vec{N}^{(1)}, \ldots, \vec{N}^{(n)}}\right|_{\omega}
$$

and

$$
|b|_{\omega^{\prime}}=\sum_{\vec{N} \in \mathbb{N}_{0}^{2}}\left|b_{\vec{N}}\right|_{\omega^{\prime}}
$$

it suffices to prove that, for any $\vec{N}^{(1)}, \ldots, \vec{N}^{(n)} \in \mathbb{N}_{0}^{2}$,

$$
\left|b_{\vec{N}^{(1)}, \ldots, \vec{N}^{(n)}}\right|_{\omega} \leq d_{1} ! \cdots d_{n} ! \prod_{j=1}^{n}\left|b_{\vec{N}^{(j)}}\right|_{\omega^{\prime}}
$$

Furthermore, since $T$ is connected, part (b) of Definition 2.3 ensures that

$$
\omega(\overrightarrow{\mathbf{x}} ; \overrightarrow{\mathbf{y}}) \leq \prod_{j=1}^{n} \omega\left(\overrightarrow{\mathbf{x}}_{j} ; \overrightarrow{\mathbf{y}}_{j}\right)
$$


for all $\overrightarrow{\mathbf{x}}_{1}, \ldots, \overrightarrow{\mathbf{x}}_{n} \in X^{(1)}$ and $\overrightarrow{\mathbf{y}}_{1}, \ldots, \overrightarrow{\mathbf{y}}_{n} \in X^{(1)}$ such that $\overrightarrow{\mathbf{x}}=\overrightarrow{\mathbf{x}}_{1} \circ \cdots \circ \overrightarrow{\mathbf{x}}_{n}, \overrightarrow{\mathbf{y}}=\overrightarrow{\mathbf{y}}_{1} \circ \cdots \circ \overrightarrow{\mathbf{y}}_{n}$ and $T \subset G\left(\overrightarrow{\mathbf{y}}_{1}, \ldots, \overrightarrow{\mathbf{y}}_{n}\right)$. So it suffices to consider $\omega=1$.

Fix any $\vec{N}^{(1)}, \ldots, \vec{N}^{(n)} \in \mathbb{N}_{0}^{2}$. Quickly review the definition (Definition 2.6) of $\left|b_{\vec{N}^{(1)}, \ldots, \vec{N}^{(n)}}\right|_{\omega}$. Fix any $\mathbf{x} \in X$ and select one component of

$$
(\overrightarrow{\mathbf{x}}, \overrightarrow{\mathbf{y}})=\left(\overrightarrow{\mathbf{x}}_{1} \circ \cdots \circ \overrightarrow{\mathbf{x}}_{n}, \overrightarrow{\mathbf{y}}_{1} \circ \cdots \circ \overrightarrow{\mathbf{y}}_{n}\right)
$$

to be anchored at $\mathbf{x}$. By permuting $\{1, \ldots, n\}$, we may assume that the one component is in $\left(\overrightarrow{\mathbf{x}}_{1}, \overrightarrow{\mathbf{y}}_{1}\right)$. For notational simplicity, we consider the case that the component is the first component $\overrightarrow{\mathbf{x}}_{1,1}$ of $\overrightarrow{\mathbf{x}}_{1}$. The other cases are virtually identical. Thus it suffices to prove that

$$
\sum_{\substack{\overrightarrow{\mathbf{y}}_{1}, \ldots, \overrightarrow{\mathbf{y}}_{n} \in X^{(1)} \\ T \subset G\left(\overrightarrow{\mathbf{y}}_{1}, \ldots, \overrightarrow{\mathbf{y}}_{n}\right)}} \sum_{\substack{\overrightarrow{\mathbf{x}}_{1}, \ldots, \vec{x}_{n} \in X^{(1)} \\ \overrightarrow{\mathbf{x}}_{1,1}=\mathbf{x}}}\left|b_{\vec{N}^{(1)}}\left(\overrightarrow{\mathbf{x}}_{1} ; \overrightarrow{\mathbf{y}}_{1}\right)\right| \cdots\left|b_{\vec{N}^{(n)}}\left(\overrightarrow{\mathbf{x}}_{n} ; \overrightarrow{\mathbf{y}}_{n}\right)\right|
$$

is bounded by $\left.d_{1} ! \cdots d_{n} ! \prod_{j=1}^{n}\left|b_{\vec{N}^{(j)}}\right|_{\omega^{\prime}}\right|_{\omega=1}$.

View 1 as the root of $T$. Then the set of vertices of $T$ is endowed with a natural partial ordering under which 1 is the smallest vertex. For each vertex $2 \leq j \leq n$, denote by $\pi(j)$ the predecessor vertex of $j$ under this partial ordering. For example, if $T$ is the tree in the figure

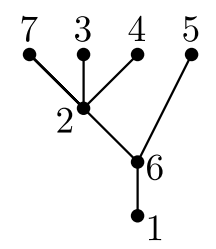

then $\pi(7)=\pi(3)=\pi(4)=2, \pi(2)=\pi(5)=6$ and $\pi(6)=1$. The condition that $T \subset$ $G\left(\overrightarrow{\mathbf{y}}_{1}, \ldots, \overrightarrow{\mathbf{y}}_{n}\right)$ ensures that, for each $2 \leq j \leq n$, the support of $\overrightarrow{\mathbf{y}}_{j}$ intersects the support of $\overrightarrow{\mathbf{y}}_{\pi(j)}$, so that at least one of the $n\left(\overrightarrow{\mathbf{y}}_{j}\right)$ components of $\overrightarrow{\mathbf{y}}_{j}$ takes the same value (in $X$ ) as some component of $\overrightarrow{\mathbf{y}}_{\pi(j)}$. Note that $n\left(\overrightarrow{\mathbf{y}}_{j}\right)=N_{2}^{(j)}$ and in particular is fixed by $\vec{N}^{(j)}$. Denote it $n_{j}$. So

$$
\text { (3.17) } \leq \prod_{j=2}^{n}\left[n_{j} n_{\pi(j)}\right] \max _{\substack{1 \leq m_{j} \leq n_{j} \\ 1 \leq p_{j} \leq n_{\pi j}(j)}} \sum_{\substack{\overrightarrow{\mathbf{y}}_{1}, \ldots, \overrightarrow{\mathbf{y}}_{n} \in X^{(1)} \\ \text { for all } 2 \leq j \leq n}} \sum_{\substack{\mathbf{y}_{j, m_{j}}=\mathbf{y}_{\pi(j), p_{j}} \\ \text { for all } 2 \leq j \leq n}} \prod_{\substack{\overrightarrow{\mathbf{x}}_{1}, \ldots, \overrightarrow{\mathbf{x}}_{n} \in X^{(1)} \\ \overrightarrow{\mathbf{x}}_{1,1}=\mathbf{x}}}^{n}\left|b_{\vec{N}^{(j)}}\left(\overrightarrow{\mathbf{x}}_{j} ; \overrightarrow{\mathbf{y}}_{j}\right)\right|
$$

Since

$$
\prod_{j=2}^{n}\left[n_{j} n_{\pi(j)}\right]=\prod_{j=1}^{n} n_{j}^{d_{j}} \leq \prod_{j=1}^{n}\left[d_{j} ! 2^{n_{j}}\right]
$$

it suffices to prove that

$$
\sum_{\substack{\overrightarrow{\mathbf{y}}_{1}, \ldots, \overrightarrow{\mathbf{y}}_{n} \in X^{(1)} \\ \overrightarrow{\mathbf{y}}_{j, m_{j}}=\overrightarrow{\mathbf{y}}_{\pi(j), p_{j}} \\ \text { for all } 2 \leq j \leq n}} \sum_{\substack{\overrightarrow{\mathbf{x}}_{1}, \ldots, \overrightarrow{\mathbf{x}}_{n} \in X^{(1)} \\ \overrightarrow{\mathbf{x}}_{1,1}=\mathbf{x}}} \prod_{j=1}^{n} 2^{n\left(\overrightarrow{\mathbf{y}}_{j}\right)}\left|b_{\vec{N}^{(j)}}\left(\overrightarrow{\mathbf{x}}_{j} ; \overrightarrow{\mathbf{y}}_{j}\right)\right| \leq\left.\prod_{j=1}^{n}\left|b_{\vec{N}^{(j)}}\right|_{\omega^{\prime}}\right|_{\omega=1}
$$


for all choices of $\left(m_{j}, p_{j}\right)_{2 \leq j \leq n}$, satisfying $1 \leq m_{j} \leq n\left(\overrightarrow{\mathbf{y}}_{j}\right)$ and $1 \leq p_{j} \leq n\left(\overrightarrow{\mathbf{y}}_{\pi(j)}\right)$. But this is done easily by iteratively applying

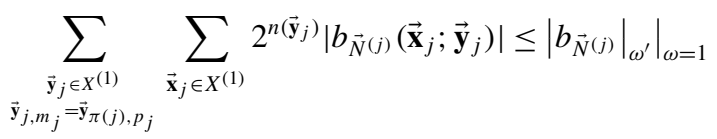

starting with the largest $j$ 's, in the partial ordering of $T$, and ending with $j=1$. (For $j=1$, substitute $\overrightarrow{\mathbf{x}}_{1,1}=\mathbf{x}$ for $\overrightarrow{\mathbf{y}}_{j, m_{j}}=\overrightarrow{\mathbf{y}}_{\pi(j), p_{j}}$.)

Lemma 3.6 Let $0<\varepsilon<\frac{1}{8}$ and $v \in \mathbb{N}$. Then

$$
\sum_{n=v}^{\infty} \frac{1}{(n-1) !} \sum_{\substack{d_{1}, \ldots, d_{n} \\
d_{1}+\cdots+d_{n}=2(n-1)}} \sum_{\begin{array}{c}
T \text { labelled tree } \\
\text { with cordination } \\
\text { numbers } d_{1}, \ldots, d_{n}
\end{array}} d_{1} ! \cdots d_{n} ! \varepsilon^{n} \leq \frac{1}{8} \frac{(8 \varepsilon)^{v}}{1-8 \varepsilon}
$$

Proof First suppose that $v \geq 2$. By the Cayley formula (see, for example [6, Theorem I.4.1]), the number of labelled trees on $n \geq 2$ vertices with specified coordination numbers $\left(d_{1}, d_{2}, \ldots, d_{n}\right)$ is

$$
\frac{(n-2) !}{\prod_{j=1}^{n}\left(d_{j}-1\right) !}
$$

The number of possible choices of coordination numbers $\left(d_{1}, d_{2}, \ldots, d_{n}\right) \in \mathbb{N}^{n}$ subject to the constraint $d_{1}+d_{2}+\cdots+d_{n}=2(n-1)$ is $\left(\begin{array}{c}2(n-1)-1 \\ n-1\end{array}\right)=\left(\begin{array}{c}2 n-3 \\ n-1\end{array}\right) \leq 2^{2 n-3}$. Therefore

$$
\begin{aligned}
& \sum_{n=\nu}^{\infty} \frac{1}{(n-1) !} \sum_{\substack{d_{1}, \ldots, d_{n} \\
d_{1}+\cdots+d_{n}=2(n-1)}} \sum_{\begin{array}{c}
T \text { labelled tree } \\
\text { with coordination } \\
\text { numbers } d_{1}, \ldots, d_{n}
\end{array}} d_{1} ! \cdots d_{n} ! \varepsilon^{n} \\
& \leq \sum_{n=v}^{\infty} \frac{1}{n-1} \sum_{\substack{d_{1}, \ldots, d_{n} \\
d_{1}+\cdots+d_{n}=2(n-1)}} d_{1} \cdots d_{n} \varepsilon^{n} \\
& \leq \sum_{n=v}^{\infty} \frac{1}{n-1} 2^{2 n-3} 2^{n} \varepsilon^{n} \leq \frac{1}{8} \frac{(8 \varepsilon)^{v}}{1-8 \varepsilon}
\end{aligned}
$$

For $n=1, d_{1}=0$ and the number of trees is 1 , so the $n=1$ term is $\varepsilon$. So the full sum for $v=1$ is bounded by $\varepsilon+\frac{1}{8} \frac{(8 \varepsilon)^{2}}{1-8 \varepsilon}=\frac{\varepsilon}{1-8 \varepsilon}$.

\section{Linear Changes of Variables and Substitutions}

In this section, we consider the effect of linear changes of variables on the norms of Definition 2.6 with the weight systems of Definition 2.5. Such changes of variables arise naturally during the course of the execution of a Wilson style renormalization group construction. For example, if $\phi_{c}$ is a critical point of the action $\mathcal{A}_{n-1}(\psi, \phi)$, it is natural to use the translation $\phi=\tilde{\phi}+\phi_{c}$; and the change of variables $\tilde{\phi}=\sqrt{C} \phi$ can be used to diagonalize the quadratic form $\sum_{\mathbf{x}, \mathbf{y}} \phi(\mathbf{x}) C(\mathbf{x}, \mathbf{y}) \phi(\mathbf{y})$. 
We fix any $m \geq 0$ and set, for a linear map $J$ from $\mathbb{R}^{X}$ to $\mathbb{R}^{X}$ with kernel $J(\mathbf{x}, \mathbf{y})$,

$$
\|\mid J\|_{m}=\max \left\{\sup _{\mathbf{x} \in X} \sum_{\mathbf{y} \in X} e^{m d(\mathbf{x}, \mathbf{y})}|J(\mathbf{x}, \mathbf{y})|, \sup _{\mathbf{y} \in X} \sum_{\mathbf{x} \in X} e^{m d(\mathbf{x}, \mathbf{y})}|J(\mathbf{x}, \mathbf{y})|\right\}
$$

For $\kappa>0$, we denote by $w_{\kappa}$ the weight system of length one with mass $m$ that associates the constant weight factor $\kappa$ to the field $\psi$. That is

$$
w_{\kappa}\left(\mathbf{x}_{1}, \ldots, \mathbf{x}_{n}\right)=e^{m \tau\left(\left\{\mathbf{x}_{1}, \ldots, \mathbf{x}_{n}\right\}\right)} \kappa^{n}
$$

Similarly, for $\kappa, \lambda>0$, we denote by $w_{\kappa, \lambda}$ the weight system of length two with mass $m$ that associates the constant weight factor $\kappa$ to the field $\psi$ and the constant weight factor $\lambda$ to the field $\phi$.

To simplify notation, we write $\|g(\psi)\|_{\kappa}$ and $\|f(\psi, \phi)\|_{\kappa, \lambda}$ for $\|g(\psi)\|_{w_{\kappa}}$ and $\|f(\psi, \phi)\|_{w_{\kappa, \lambda}}$, respectively.

Proposition 1 Let $g$ be an analytic function on a neighbourhood of the origin in $\mathbb{R}^{X}$.

(i) Let $J$ be an operator on $\mathbb{R}^{X}$ with kernel $J(\mathbf{x}, \mathbf{y})$. Define $\tilde{g}$ by

$$
\tilde{g}(\psi)=g(J \psi)
$$

Let $\kappa>0$ and set $\kappa^{\prime}=\kappa\|J \mid\|_{m}$. Then $\|\tilde{g}\|_{\kappa} \leq\|g\|_{\kappa^{\prime}}$.

(ii) Define $f$ by

$$
f(\psi ; \phi)=g(\psi+\phi)
$$

Then $\|f\|_{\kappa, \lambda}=\|g\|_{\kappa+\lambda}$.

Proof (i) Let $a(\overrightarrow{\mathbf{x}})$ be a symmetric coefficient system for $g$. Define, for each $n \geq 0$,

$$
\tilde{a}\left(\mathbf{x}_{1}, \ldots, \mathbf{x}_{n}\right)=\sum_{\mathbf{y}_{1}, \ldots, \mathbf{y}_{n} \in X} a\left(\mathbf{y}_{1}, \ldots, \mathbf{y}_{n}\right) \prod_{\ell=1}^{n} J\left(\mathbf{y}_{\ell}, \mathbf{x}_{\ell}\right)
$$

Then $\tilde{a}(\overrightarrow{\mathbf{x}})$ is a symmetric coefficient system for $\tilde{g}$. Since

$$
\tau\left(\left\{\mathbf{x}_{1}, \ldots, \mathbf{x}_{n}\right\}\right) \leq \tau\left(\left\{\mathbf{y}_{1}, \ldots, \mathbf{y}_{n}\right\}\right)+\sum_{\ell=1}^{n} d\left(\mathbf{y}_{\ell}, \mathbf{x}_{\ell}\right)
$$

we have

$$
e^{m \tau\left(\left\{\mathbf{x}_{1}, \ldots, \mathbf{x}_{n}\right\}\right)} \leq e^{m \tau\left(\left\{\mathbf{y}_{1}, \ldots, \mathbf{y}_{n}\right\}\right)} \prod_{\ell=1}^{n} e^{m d\left(\mathbf{y}_{\ell}, \mathbf{x}_{\ell}\right)}
$$

and hence

$$
\begin{aligned}
& w_{\kappa}\left(\mathbf{x}_{1}, \ldots, \mathbf{x}_{n}\right)\left|\tilde{a}\left(\mathbf{x}_{1}, \ldots, \mathbf{x}_{n}\right)\right| \\
& \quad \leq \sum_{\mathbf{y}_{1}, \ldots, \mathbf{y}_{n} \in X} w_{\kappa^{\prime}}\left(\mathbf{y}_{1}, \ldots, \mathbf{y}_{n}\right)\left|a\left(\mathbf{y}_{1}, \ldots, \mathbf{y}_{n}\right)\right| \prod_{\ell=1}^{n}\left[\frac{\kappa}{\kappa^{\prime}} e^{m d\left(\mathbf{y}_{\ell}, \mathbf{x}_{\ell}\right)}\left|J\left(\mathbf{y}_{\ell}, \mathbf{x}_{\ell}\right)\right|\right]
\end{aligned}
$$


We are to bound

$$
\begin{aligned}
\|\tilde{g}\|_{\kappa}= & \sum_{n \geq 0} \max _{\mathbf{x} \in X} \max _{1 \leq j \leq n} \sum_{\substack{\mathbf{x}_{1}, \ldots, \mathbf{x}_{n} \in X^{n} \\
\mathbf{x}_{j}=\mathbf{x}}} w_{\kappa}\left(\mathbf{x}_{1}, \ldots, \mathbf{x}_{n}\right)\left|\tilde{a}\left(\mathbf{x}_{1}, \ldots, \mathbf{x}_{n}\right)\right| \\
\leq & \sum_{n \geq 0} \max _{\mathbf{x} \in X} \max _{1 \leq j \leq n} \sum_{\substack{\mathbf{x}_{1}, \ldots, \mathbf{x}_{n} \in X \\
\mathbf{x}_{j}=\mathbf{x}}} \sum_{\mathbf{y}_{1}, \ldots, \mathbf{y}_{n} \in X} w_{\kappa^{\prime}}\left(\mathbf{y}_{1}, \ldots, \mathbf{y}_{n}\right)\left|a\left(\mathbf{y}_{1}, \ldots, \mathbf{y}_{n}\right)\right| \\
& \times \prod_{\ell=1}^{n}\left[\frac{\kappa}{\kappa^{\prime}} e^{m d\left(\mathbf{y}_{\ell}, \mathbf{x}_{\ell}\right)}\left|J\left(\mathbf{y}_{\ell}, \mathbf{x}_{\ell}\right)\right|\right]
\end{aligned}
$$

Fix any $n \geq 0, \mathbf{x} \in X$ and $1 \leq j \leq n$. By definition, for each $\ell \neq j$ and $\mathbf{y}_{\ell} \in X$

$$
\sum_{\mathbf{x}_{\ell} \in X} \frac{\kappa}{\kappa^{\prime}} e^{m d\left(\mathbf{y}_{\ell}, \mathbf{x}_{\ell}\right)} J\left(\mathbf{y}_{\ell}, \mathbf{x}_{\ell}\right)=\sum_{\mathbf{x}_{\ell} \in X} \frac{1}{\|J\|_{m}} e^{m d\left(\mathbf{y}_{\ell}, \mathbf{x}_{\ell}\right)} J\left(\mathbf{y}_{\ell}, \mathbf{x}_{\ell}\right) \leq 1
$$

Therefore

$$
\begin{aligned}
& \sum_{\substack{\mathbf{x}_{1}, \ldots, \mathbf{x}_{n} \in X \\
\mathbf{x}_{j}=\mathbf{x}}} \sum_{\substack{\mathbf{y}_{1}, \ldots, \mathbf{y}_{n} \in X\\
}} w_{\kappa^{\prime}}\left(\mathbf{y}_{1}, \ldots, \mathbf{y}_{n}\right)\left|a\left(\mathbf{y}_{1}, \ldots, \mathbf{y}_{n}\right)\right| \prod_{\ell=1}^{n}\left[\frac{\kappa}{\kappa^{\prime}} e^{m d\left(\mathbf{y}_{\ell}, \mathbf{x}_{\ell}\right)}\left|J\left(\mathbf{y}_{\ell}, \mathbf{x}_{\ell}\right)\right|\right] \\
& \leq \sum_{\mathbf{y} \in X} \frac{\kappa}{\kappa^{\prime}} e^{m d(\mathbf{y}, \mathbf{x})} J(\mathbf{y}, \mathbf{x}) \sum_{\substack{\mathbf{y}_{1}, \ldots, \mathbf{y}_{n} \in X \\
\mathbf{y}_{j}=\mathbf{y}}} w_{\kappa^{\prime}}\left(\mathbf{y}_{1}, \ldots, \mathbf{y}_{n}\right)\left|a\left(\mathbf{y}_{1}, \ldots, \mathbf{y}_{n}\right)\right| \\
& \leq \sum_{\mathbf{y} \in X} \frac{\kappa}{\kappa^{\prime}} e^{m d(\mathbf{y}, \mathbf{x})} J(\mathbf{y}, \mathbf{x}) \max _{\mathbf{y} \in X} \sum_{\substack{\mathbf{y}_{1}, \ldots, \mathbf{y}_{n} \in X \\
\mathbf{y}_{j}=\mathbf{y}}} w_{\kappa^{\prime}}\left(\mathbf{y}_{1}, \ldots, \mathbf{y}_{n}\right)\left|a\left(\mathbf{y}_{1}, \ldots, \mathbf{y}_{n}\right)\right| \\
& \leq \max _{\mathbf{y} \in X} \sum_{\substack{\mathbf{y}_{1}, \ldots, \mathbf{y}_{n} \in X \\
\mathbf{y}_{j}=\mathbf{y}}} w_{\kappa^{\prime}}\left(\mathbf{y}_{1}, \ldots, \mathbf{y}_{n}\right)\left|a\left(\mathbf{y}_{1}, \ldots, \mathbf{y}_{n}\right)\right|
\end{aligned}
$$

since, once again, $\sum_{\mathbf{y} \in X} \frac{\kappa}{\kappa^{\prime}} e^{m d(\mathbf{y}, \mathbf{x})} J(\mathbf{y}, \mathbf{x})=\sum_{y \in X} \frac{1}{\|J\|_{m}} e^{m d(\mathbf{y}, \mathbf{x})} J(\mathbf{y}, \mathbf{x}) \leq 1$. Consequently, (4.1) is bounded by

$$
\sum_{n \geq 0} \max _{1 \leq j \leq n} \max _{\mathbf{y} \in X} \sum_{\substack{\mathbf{y}_{1}, \ldots, \mathbf{y}_{n} \in X \\ \mathbf{y}_{j}=\mathbf{y}}} w_{\kappa^{\prime}}\left(\mathbf{y}_{1}, \ldots, \mathbf{y}_{n}\right)\left|a\left(\mathbf{y}_{1}, \ldots, \mathbf{y}_{n}\right)\right|=\|g\|_{\kappa^{\prime}}
$$

This proves part (i) of the proposition.

(ii) Let $a(\overrightarrow{\mathbf{u}})$ be a symmetric coefficient system for $g$. Since $a$ is invariant under permutation of its $\overrightarrow{\mathbf{u}}$ components,

$$
g(\psi+\phi)=\sum_{\overrightarrow{\mathbf{u}} \in \mathbf{X}^{(1)}} a(\overrightarrow{\mathbf{u}})(\psi+\phi)(\overrightarrow{\mathbf{u}})=\sum_{\overrightarrow{\mathbf{x}}, \overrightarrow{\mathbf{y}} \in \mathbf{X}^{(1)}} a(\overrightarrow{\mathbf{x}} \circ \overrightarrow{\mathbf{y}})\left(\begin{array}{c}
n(\overrightarrow{\mathbf{x}})+n(\overrightarrow{\mathbf{y}}) \\
n(\overrightarrow{\mathbf{y}})
\end{array}\right) \psi(\overrightarrow{\mathbf{x}}) \phi(\overrightarrow{\mathbf{y}})
$$

so that

$$
a_{+}(\overrightarrow{\mathbf{x}} ; \overrightarrow{\mathbf{y}})=a(\overrightarrow{\mathbf{x}} \circ \overrightarrow{\mathbf{y}})\left(\begin{array}{c}
n(\overrightarrow{\mathbf{x}})+n(\overrightarrow{\mathbf{y}}) \\
n(\overrightarrow{\mathbf{y}})
\end{array}\right)
$$


is a symmetric coefficient system for $f$. We have

$$
\begin{aligned}
\|f\|_{\kappa, \lambda} & =\sum_{k, \ell \geq 0} \max _{\mathbf{p} \in X} \max _{1 \leq i \leq k+\ell} \sum_{\substack{\overrightarrow{\mathbf{x}} \in X^{k}, \overrightarrow{\mathbf{y}} \in X^{\ell} \\
(\overrightarrow{\mathbf{x}}, \mathbf{y})}} w_{\kappa, \lambda}(\overrightarrow{\mathbf{x}} ; \overrightarrow{\mathbf{y}})\left|a_{+}(\overrightarrow{\mathbf{x}} ; \overrightarrow{\mathbf{y}})\right| \\
& =\sum_{k, \ell \geq 0} \max _{\mathbf{p} \in X} \max _{1 \leq i \leq k+\ell} \sum_{\substack{\overrightarrow{\mathbf{x}} \in X^{k}, \overrightarrow{\mathbf{y}} \in X^{\ell} \\
(\overrightarrow{\mathbf{x}}, \overrightarrow{\mathbf{y}})_{i}=\mathbf{p}}} e^{m \tau(\operatorname{supp}(\overrightarrow{\mathbf{x}}, \overrightarrow{\mathbf{y}}))} \kappa^{k} \lambda^{\ell}\left(\begin{array}{c}
k+\ell \\
\ell
\end{array}\right)|a(\overrightarrow{\mathbf{x}} \circ \overrightarrow{\mathbf{y}})| \\
& =\sum_{k, \ell \geq 0}\left(\begin{array}{c}
k+\ell \\
\ell
\end{array}\right) \kappa^{k} \lambda^{\ell} \max _{\mathbf{p} \in X} \max _{1 \leq i \leq k+\ell} \sum_{\substack{\overrightarrow{\mathbf{x}} \in X^{k}, \overrightarrow{\mathbf{y}} \in X^{\ell} \\
(\mathbf{x}, \vec{y})}} e_{i=\mathbf{p}}^{m \tau(\operatorname{supp}(\overrightarrow{\mathbf{x}}, \overrightarrow{\mathbf{y}}))}|a(\overrightarrow{\mathbf{x}} \circ \overrightarrow{\mathbf{y}})| \\
& =\sum_{k, \ell \geq 0}\left(\begin{array}{c}
k+\ell \\
\ell
\end{array}\right) \kappa^{k} \lambda^{\ell} \max _{\mathbf{p} \in X} \max _{1 \leq i \leq k+\ell} \sum_{\substack{\overrightarrow{\mathbf{u}} \in X^{k+\ell} \\
\mathbf{u}_{i}=\mathbf{p}}} e^{m \tau(\operatorname{supp}(\overrightarrow{\mathbf{u}}))}|a(\overrightarrow{\mathbf{u}})| \\
& =\sum_{n \geq 0}(\kappa+\lambda)^{n} \max _{\mathbf{p} \in X} \max _{1 \leq i \leq n} \sum_{\substack{\mathbf{u} \in X^{n} \\
\overrightarrow{\mathbf{u}} i=\mathbf{p}}} e^{m \tau(\operatorname{supp}(\overrightarrow{\mathbf{u}}))}|a(\overrightarrow{\mathbf{u}})| \\
& =\|g\|_{\kappa+\lambda}
\end{aligned}
$$

\section{References}

1. Balaban, T., Feldman, J., Knörrer, H., Trubowitz, E.: A functional integral representation for many boson systems. I: The partition function. Ann. Henri Poincaré 9, 1229-1273 (2008)

2. Balaban, T., Feldman, J., Knörrer, H., Trubowitz, E.: A functional integral representation for many boson systems. II: Correlation functions. Ann. Henri Poincaré 9, 1275-1307 (2008)

3. Balaban, T., Feldman, J., Knörrer, H., Trubowitz, E.: Power series representations for complex bosonic effective actions (2008, preprint)

4. Balaban, T., Feldman, J., Knörrer, H., Trubowitz, E.: Power series representations for complex bosonic effective actions. II. A small field renormalization group flow (2008, preprint)

5. Cammarota, C.: Decay of correlations for infinite range interactions in unbounded spin systems. Commun. Math. Phys. 85, 517-528 (1982)

6. Rivasseau, V.: From Perturbative to Constructive Renormalization. Princeton University Press, Princeton (1991)

7. Rota, G.-C.: On the foundations of combinatorial theory. I. Theory of Möbius functions. Z. Wahrscheinlichkeitstheor. Verw. Geb. 2, 340 (1964)

8. Salmhofer, M.: Renormalization, An Introduction. Springer, Berlin (1999)

9. Seiler, E.: Gauge Theories as a Problem of Constructive Quantum Field Theory and Statistical Mechanics. Springer, Berlin (1982)

10. Simon, B.: The Statistical Mechanics of Lattice Gases, vol. 1. Princeton University Press, Princeton (1993) 